April 6, 2010 - final version for proceedings of CiE'10

\title{
What is the Problem with Proof Nets for Classical Logic?
}

\author{
Lutz Straßburger \\ INRIA Saclay - Île-de-France and LIX, École Polytechnique \\ http://www.lix.polytechnique.fr/ lutz
}

\begin{abstract}
This paper is an informal (and nonexhaustive) overview over some existing notions of proof nets for classical logic, and gives some hints why they might be considered to be unsatisfactory.
\end{abstract}

\section{Introduction}

There is a very close and well-understood relationship between proofs in intuitionistic logic, simply typed lambda-terms, and morphisms in Cartesian closed categories. The same relationship can be established for multiplicative linear logic (MLL), where proof nets take the role of the lambda-terms, and starautonomous categories the role of Cartesian closed categories.

It is certainly desirable to have something similar for classical logic, which can be obtained from intuitionistic logic by adding the law of excluded middle, i.e., $A \vee \bar{A}$, or equivalently, an involutive negation, i.e., $\overline{\bar{A}}=A$. Adding this to a Cartesian closed category $\mathscr{C}$, means adding a contravariant functor $\overline{(-)}: \mathscr{C} \rightarrow \mathscr{C}$ such that $\overline{\bar{A}} \cong A$ and $\overline{(A \wedge B)} \cong \bar{A} \vee \bar{B}$ where $A \vee B=\bar{A} \Rightarrow B$. However, if we do this we get a collapse: all proofs of the same formula are identified, which leads to a rather boring proof theory. This observation is due to André Joyal, and a proof and discussion can be found in 123 .

Here we will not show the category theoretic proof of the collapse, but will quickly explain the phenomenon in terms of the sequent calculus (the argumentation is due to Yves Lafont [4, Appendix B]). Suppose we have two proofs $\Pi_{1}$ and $\Pi_{2}$ of a formula $B$ in some sequent calculus system. Then we can form, with the help of the rules weakening, contraction, and cut, the following proof of $B$ :

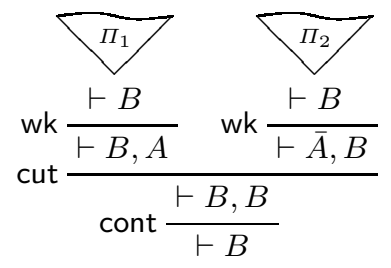

If we apply cut elimination to this proof, we get either 


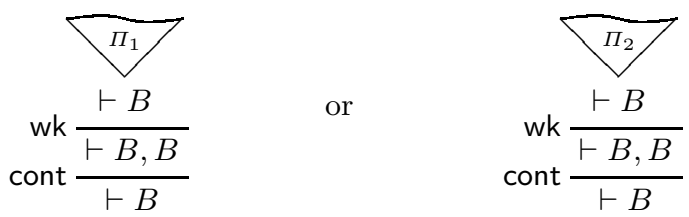

depending on a nondeterministic choice. On the other hand, if we want the nice relationship between deductive system and category theory, we need a confluent cut elimination, which means that the two proofs in (2) must be the same. Consequently, we have to equate $\Pi_{1}$ and $\Pi_{2}$. Since there was no initial condition on $\Pi_{1}$ and $\Pi_{2}$, we conclude that any two proofs of $B$ must be equal.

The problem with weakening, which could in fact be solved by using the mix-rule

$$
\operatorname{mix} \frac{\vdash \Gamma \quad \vdash \Delta}{\vdash \Gamma, \Delta},
$$

is not the only one. We run into similar problems with the contraction rule. If we try to eliminate the cut from

$$
\operatorname{cont} \frac{\vdash \Gamma, A, A}{\vdash \Gamma, A} \operatorname{cont} \frac{\vdash \bar{A}, \bar{A}, \Delta}{\vdash \bar{A}, \Delta} .
$$

we again have to make a nondeterministic choice. In Section 2 we will see a concrete example for this.

There are several possibilities to cope with these problems. Clearly, we have to drop some of the equations that we would like to hold between proofs in classical logic. But which ones should go?

There are now essentially three different approaches, and all three have their advantages and disadvantages.

1. The first says that the axioms of Cartesian closed categories are essential and cannot be dispensed with. Instead, one sacrifices the duality between $\wedge$ and $\vee$. The motivation for this approach is that a proof system for classical logic can now be seen as an extension of the $\lambda$-calculus and the notion of normalization does not change. One has a term calculus for proofs, namely Parigot's $\lambda \mu$-calculus [5] and a denotational semantics [2]. An important aspect is the computational meaning in terms of continuations 67. There is a well explored category theoretical axiomatization [8, and, of course, a theory of proof nets 9 , which is based on the proof nets for multiplicative exponential linear logic (MELL).

2. The second approach considers the perfect symmetry between $\wedge$ and $\vee$ to be an essential facet of Boolean logic, that cannot be dispensed with. Consequently, the axioms of Cartesian closed categories and the close relation to the $\lambda$-calculus have to be sacrificed. More precisely, the conjunction $\wedge$ is no longer a Cartesian product, but merely a tensor-product. Thus, the Cartesian closed structure is replaced by a star-autonomous structure, as it it known 
from linear logic. However, the precise category theoretical axiomatization is much less clear than in the first approach (see [101112313]).

3 . The third approach keeps the perfect symmetry between $\wedge$ and $\vee$, as well as the Cartesian product property for $\wedge$. What has to be dropped is the property of being closed, i.e., there is no longer a bijection between the proofs of

$$
A \vdash B \Rightarrow C \quad \text { and } \quad A \wedge B \vdash C \quad,
$$

which means we lose currying. This approach is studied in [14.

In this paper, we only focus on the second approach. The various notions of proof nets that fall in this setting can be grouped into two different ideologies:

Sequent Rule Ideology: A proof net is a graph in which every vertex represents an inference rule application in the corresponding sequent calculus proof, and every edge of the graph stands for a formula appearing in the proof. A sequent calculus proof with conclusion $\vdash A_{1}, A_{2}, \ldots, A_{n}$, written as

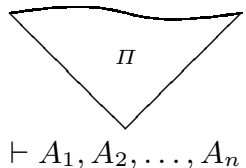

is translated into a proof net with conclusions $A_{1}, A_{2}, \ldots, A_{n}$, written as

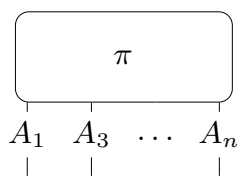

Flow Graph Ideology: A proof net consists of the formula tree/sequent forest of the conclusion of the proof, together with some additional graph structure capturing the "essence" of the proof (whatever that means).

It should be observed that for multiplicative linear logic (MLL) the two ideologies produce the same notion of proof nets. However, for classical logic the situation is very different.

\section{Sequent calculus rule based proof nets}

The set of formulas is defined via

$$
\mathscr{F}::=\mathscr{A}|\overline{\mathscr{A}}| \mathscr{F} \vee \mathscr{F} \mid \mathscr{F} \wedge \mathscr{F}
$$

where $\mathscr{A}=\{a, b, c, \ldots\}$ is a countable set of propositional variables, and $\mathscr{A}=$ $\{\bar{a}, \bar{b}, \bar{c}, \ldots\}$ are their duals. In the following, the elements of the set $\mathscr{A} \cup \mathscr{A}$ are called atoms. The negation ${ }^{-}$is defined via DeMorgan laws for all formulas. For simplicity, we ignore the units and push negation to the atoms. There are various 
different sequent calculus systems for classical propositional logic, starting with the one by Gentzen [15]. Figure 1 shows on the top (first and third line) the one we use here (for simplicity, we use a one-sided system). As indicated by Girard [2, and detailed out by Robinson [16, any sequent proof $\Pi$ can (inductively) be translated into a proof net $\pi$, as shown on the bottom (second and forth line) of Figure 1. A proof net is then a graph whose vertices are the rule instances of $\Pi$, and whose edges are labeled by the principal formulas of the rules. For simplicity, we consider the outputs to be unordered and ignore the exchange rule. Here is an example of a sequent calculus proof

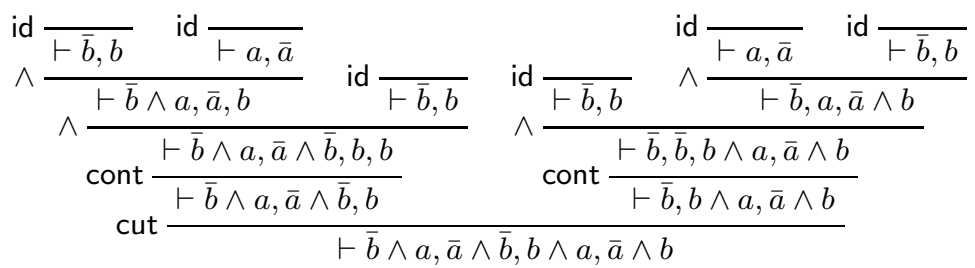

and its translation into a proof net:

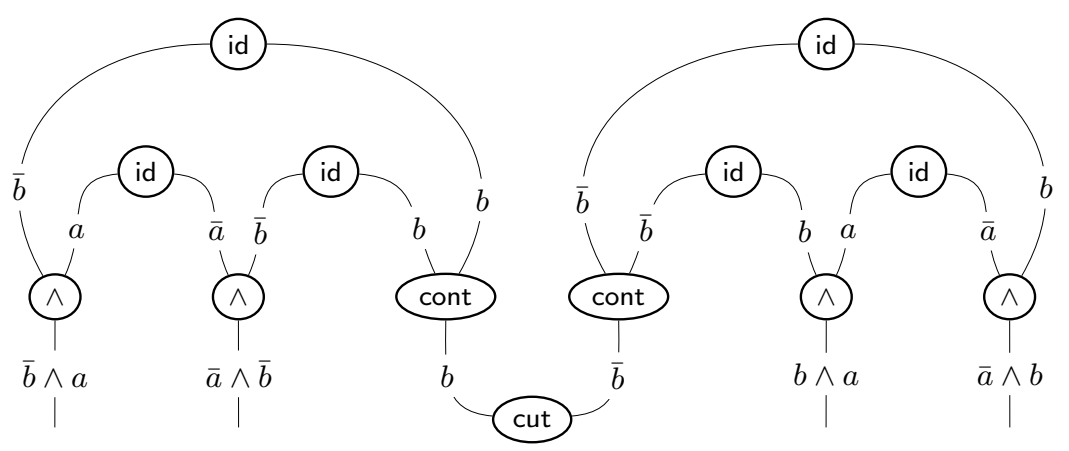

The advantage of the sequent-rule-ideology is that all the correctness criteria for MLL proof nets hold unchanged. For example, the switching criterion [17] A (well-formed) proof net is the translation of a sequent proof if and only if each of its switchings is a connected and acyclic graph, where a switching of a proof net $\pi$ is a graph that is obtained from $\pi$ by removing for each $\vee$-node and each cont-node one of the two edges connecting it to its children.

There are however two main disadvantages of the sequent-rule-ideology. The first is that certain proofs are distinguished that should be identified according the rule-permutability-argument. To see a very simple example, consider the following three sequent calculus proofs:

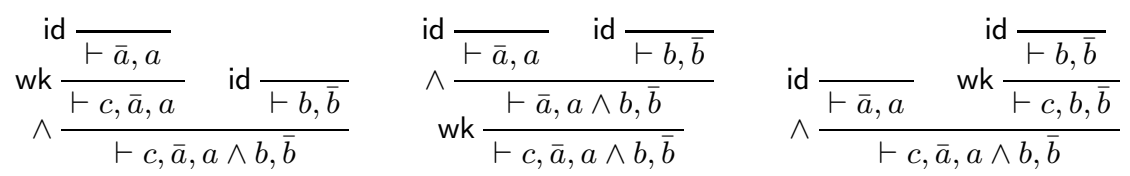

They differ from each other only via some trivial rule permutation, and should therefore be identified. But they can be translated into five different proof nets. 
id $\frac{}{\vdash \bar{A}, A}$

$\downarrow$
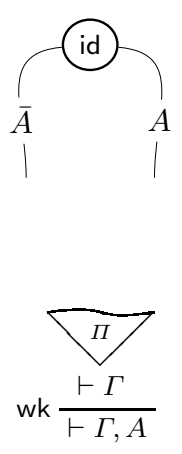

$\downarrow$

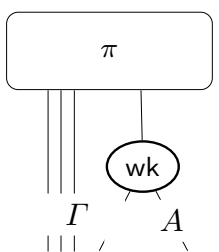

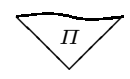

$\vee \frac{\vdash \Gamma, A, B}{\vdash \Gamma, A \vee B}$

$\downarrow$
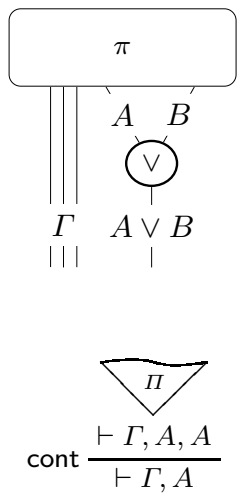

$\downarrow$

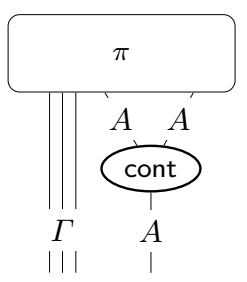

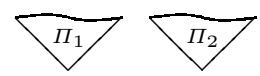

$\wedge \frac{\vdash \Gamma, A \quad \vdash B, \Delta}{\vdash \Gamma, A \wedge B, \Delta}$

$\downarrow$
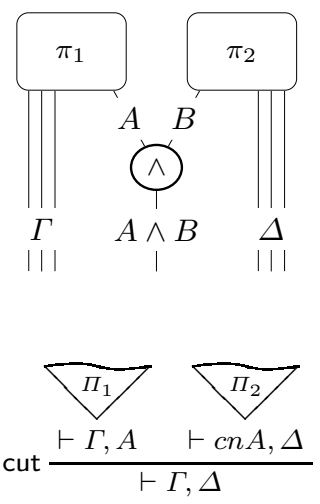

$\downarrow$

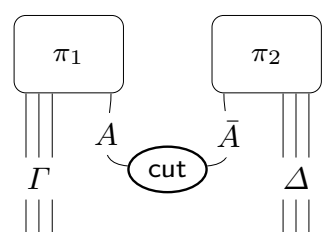

Fig. 1. From sequent calculus to proof nets (sequent rule ideology)

Two of them are shown below:
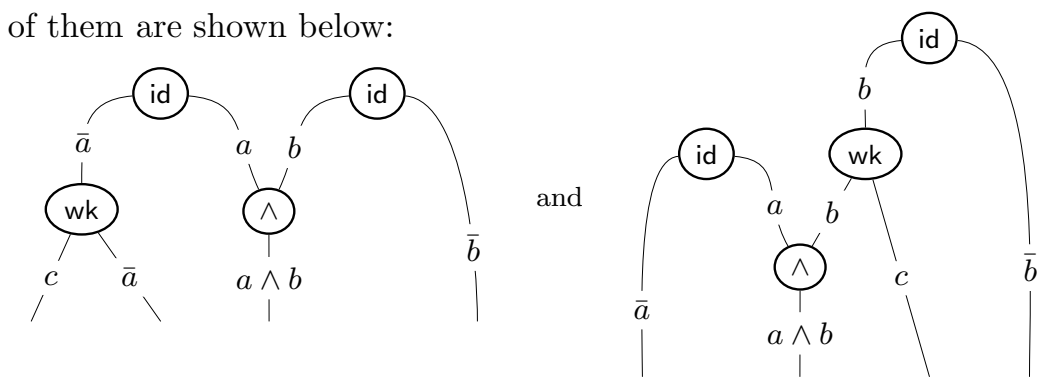

The problem is that there is no canonical choice of where to attach the weakening. A possible solution could be to leave the weakenings unconnected, but this would break the correctness criteria.

The second disadvantage of the sequent-rule-ideology is related to cut elimination. In the introduction we have seen already the problem with weakening. Let us now have a closer look at contraction, when it appears at both sides of a 


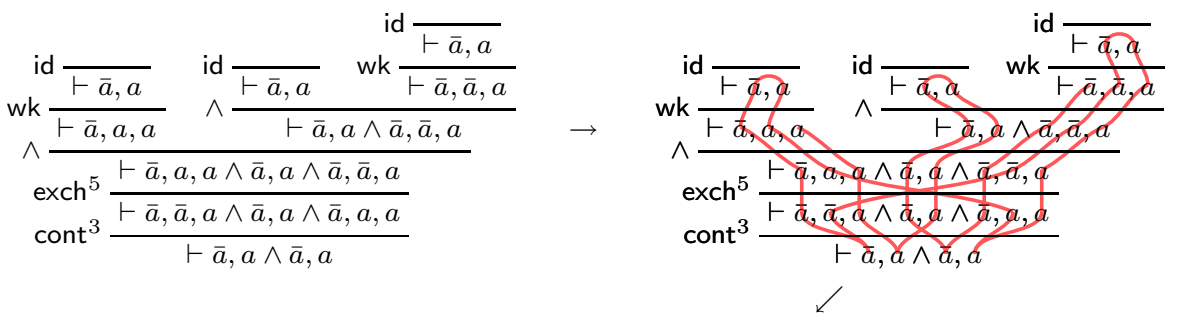

(B-net)
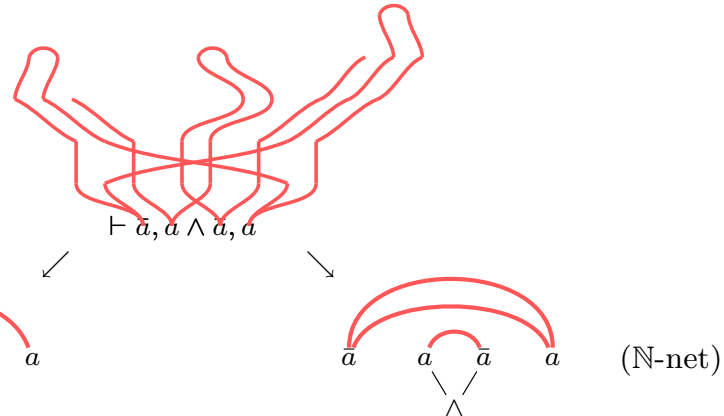

Fig. 2. From sequent calculus to proof nets (flow graph ideology)

cut, as shown in the example in (6) and (7). For typesetting reasons, let us use the more compact notation:

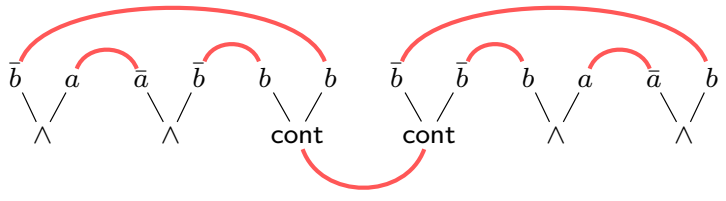

We have here an example for the general case in (4). If we want to eliminate the cut from (10), we have to make a nondeterministic choice, which subproof we duplicate. As outcome we get either

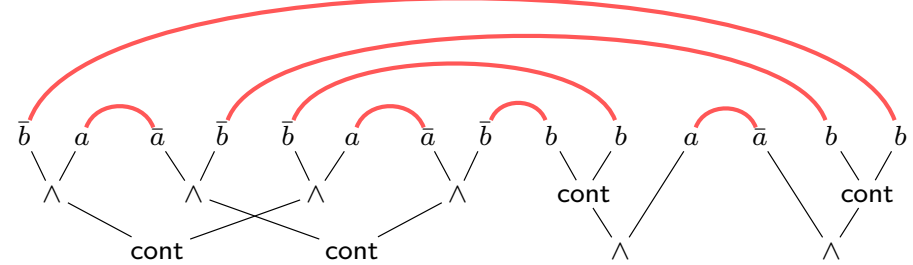

or

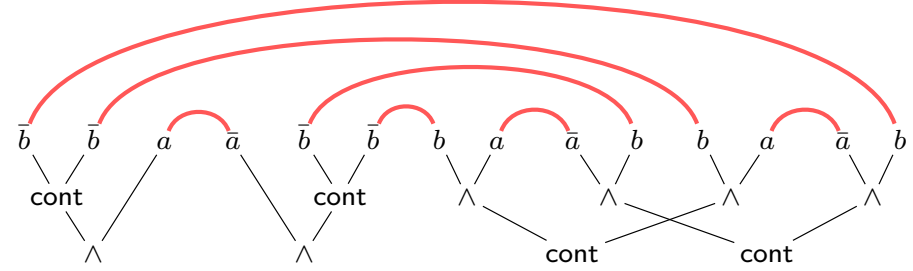


In 2, Girard argues that for this reason it is impossible to have a confluent notion of cut elimination for proof nets for classical logic. Of course, his argumentation is valid only for proof nets following the sequent-rule-ideology.

Thus, for changing the situation with cut elimination, one has to change the ideology.

\section{Flow graph based proof nets}

The basic idea is to draw the flow graph 18 of the proof as indicated in Figure 2 The important question is what information should be kept. In 19 there are two proposals. The first takes the sequent forest and adds an edge between a pair of dual atoms if they are connected by a path in the flow graph. This yields the $\mathbb{B}$-nets of [19], and an example is shown on the lower left of Figure 2] The second approach also keeps the number of paths between two atoms. The result is called $\mathbb{N}$-nets in [19]. This is denoted by either labeling the edge between the two atoms by a natural number, or by drawing multiple edges, as shown on the lower right of Figure 2 In both cases it can happen that some atoms have no mate, i.e., live celibate, and that some atoms have more than one mate, i.e., live polygamous. This is the main difference to MLL proof nets, where every single atom lives monogamous.

Cuts are shown as special edges between the roots of the formula trees, as in this example, which is obtained from the sequent proof in [6]:

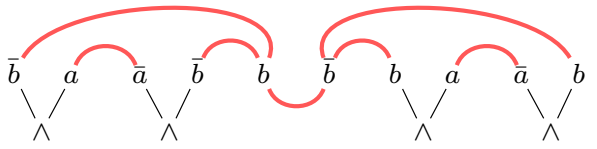

The disadvantage of the flow-graph-ideology is that the correctness criteria from linear logic are no longer available. However, for $\mathbb{B}$-nets there is a correctness criterion that is similar to the criterion for matings [20] and matrix proofs [21]: $A \mathbb{B}$-net is the translation of a sequent proof if and only if each of its conjunctive prunings contains at least one axiom link edge, where a conjunctive pruning for $\pi$ is obtained from $\pi$ by deleting for each of its $\wedge$-nodes one of the two subtrees including the outgoing axiom link edges.

The main problem with of this criterium is that checking it takes exponential time in the size of the input. This means that checking a given proof is as expensive as finding the proof from scratch.

Furthermore, this criterion does not work for $\mathbb{N}$-nets because it does not take into account how often an axiom link edge is used, and it is an open problem to find some correctness criterion for $\mathbb{N}$-nets.

Let us now look at cut elimination. Reducing cuts on compound formulas is exactly the same as in the previous section:

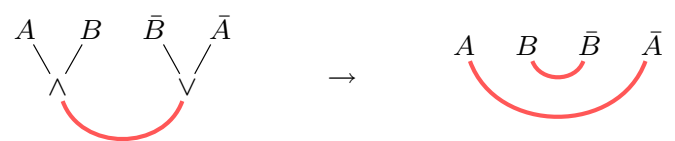


For the cut reduction on atomic cuts, we have to be careful, since the atoms can be connected to many other atoms (or no other atoms). Instead of simply having:

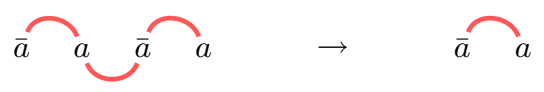

as in MLL, the reduction looks as follows:

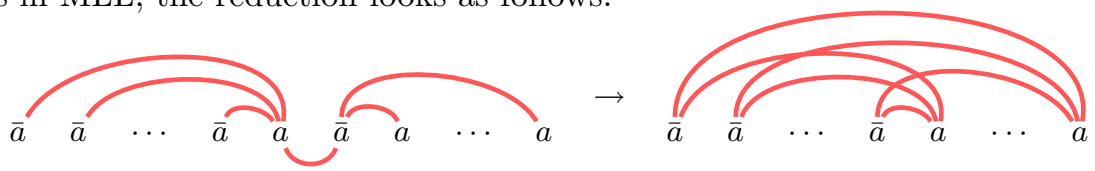

If one of the two cut atoms is celibate, no link remains:

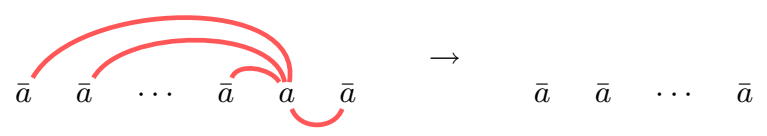

If the two cut atoms are linked together, then this link is ignored in the reduction (and, of course, removed with the cut):

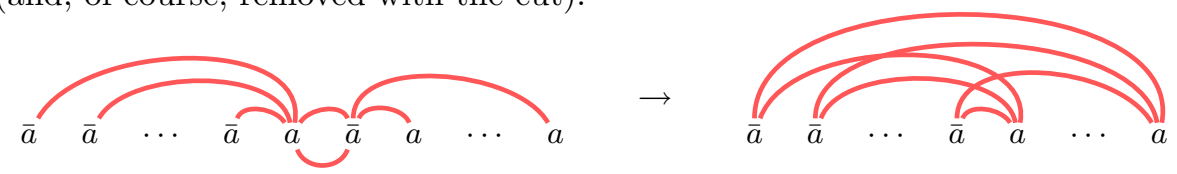

We certainly have termination of the cut reduction. The interesting observation is that for $\mathbb{B}$-nets, the cut reduction preserves correctness and is confluent.

The natural question that arises now is: How does this confluent cut elimination relate to the non-confluent cut elimination in the sequent calculus?

Let us look again at the two problematic cases (11) and (4). The problem with weakening (11) can easily be solved by using the mix-rule in the sequent calculus:

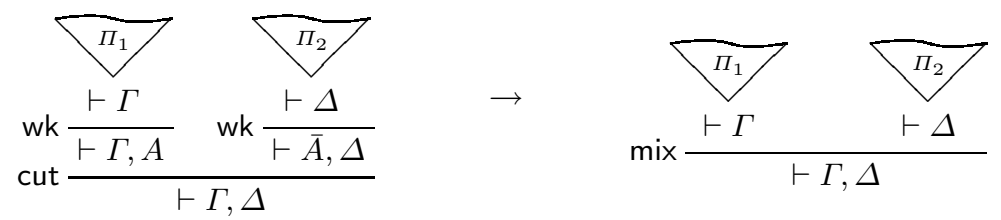

Both subproofs $\Pi_{1}$ and $\Pi_{2}$ are kept in the reduced net, and in $\mathbb{B}$-nets and $\mathbb{N}$-nets it is done in the same way.

For the contraction case (4) the situation is less obvious. Consider again the simple proof net in (13), which corresponds to the sequent calculus proof in (6). If we apply the cut reduction (16), we obtain the following result:

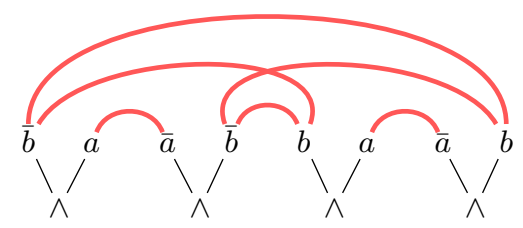

which is exactly the $\mathbb{B}$-net obtained from the sequent proofs corresponding to (11) and (12). This correspondence makes crucial use of the fact that we deliberately 
forget how often an identity link is used in the proof. As $\mathbb{N}$-nets, the proofs in (11) and (12) would be represented by
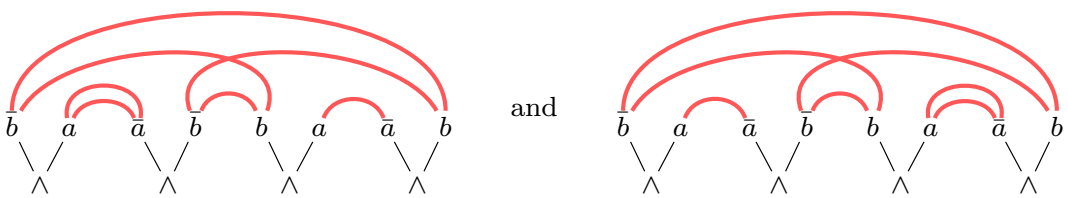

respectively (see 19] for further details). However, although it is not possible to have (18) as $\mathbb{N}$-net of a sequent proof, it can be obtained as a $\mathbb{N}$-net of a proof in the calculus of structures [22], more precisely in system SKS, as presented in 23]:

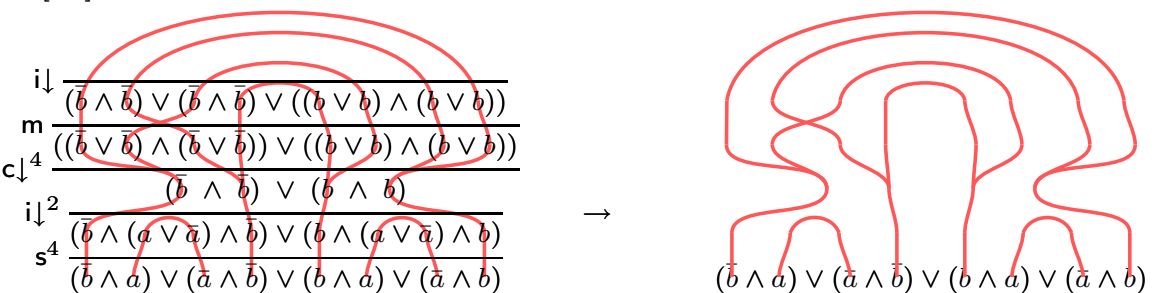

This means that any correctness criterion for $\mathbb{N}$-nets must depend on the chosen deductive system.

The non-confluence of cut-reduction for $\mathbb{N}$-nets has the following reason. When we reduce an atomic cut, we have to multiply the number of edges, and if there are already some links between the remaining pair of atoms, then these links have to be added. For example

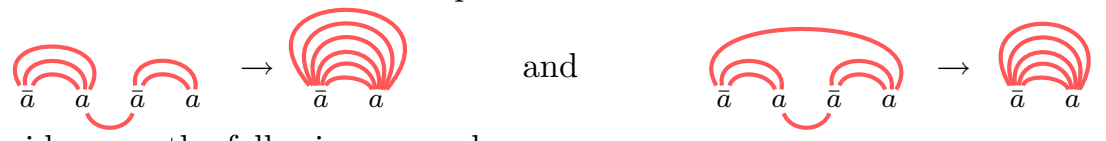

Consider now the following example:

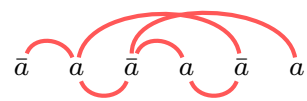

Depending on which cut we reduce first, we get either

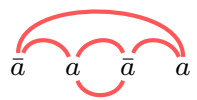

or

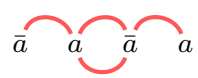

If we reduce the remaining cut, we get

$$
\overbrace{\bar{a}} \text { or } \quad \widehat{a} a
$$

respectively. The solution for circumventing this problem is to reduce atomic cuts only in unproblematic situations like (15) and (17), and leave all atomic cuts like (16) unreduced, as it is done for C-nets in 24. C-nets are a variant of $\mathbb{N}$-nets that are considered cut-free if they contain only atomic cuts that touch a contraction on both sides. In this way C-nets can also capture the size of a proof, because the reduction (16) is the only one which causes an exponential blow-up of the proof. C-nets can also be used as coherence graphs for SKS-derivations. The same approach is taken by the recently developed atomic flows [25]. 


\section{References}

1. Lambek, J., Scott, P.J.: Introduction to higher order categorical logic. Volume 7 of Cambridge studies in advanced mathematics. Cambridge University Press (1986)

2. Girard, J.Y.: A new constructive logic: Classical logic. Math. Structures in Comp. Science 1 (1991) 255-296

3. Straßburger, L.: On the axiomatisation of Boolean categories with and without medial. Theory and Applications of Categories 18(18) (2007) 536-601

4. Girard, J.Y., Lafont, Y., Taylor, P.: Proofs and Types. Cambridge Tracts in Theoretical Computer Science. Cambridge University Press (1989)

5. Parigot, M.: $\lambda \mu$-calculus: An algorithmic interpretation of classical natural deduction. In: LPAR 1992. Volume 624 of LNAI., Springer-Verlag (1992) 190-201

6. Thielecke, H.: Categorical Structure of Continuation Passing Style. PhD thesis, University of Edinburgh (1997)

7. Streicher, T., Reus, B.: Classical logic, continuation semantics and abstract machines. J. of Functional Programming 8(6) (1998) 543-572

8. Selinger, P.: Control categories and duality: on the categorical semantics of the lambda-mu calculus. Math. Structures in Comp. Science 11 (2001) 207-260

9. Laurent, O.: Polarized proof-nets and $\lambda \mu$-calculus. Theoretical Computer Science 290(1) (2003) 161-188

10. Führmann, C., Pym, D.: Order-enriched categorical models of the classical sequent calculus. To appear in J. of Pure and Applied Algebra (2004)

11. Lamarche, F., Straßburger, L.: Constructing free Boolean categories. In: LICS'05. (2005) 209-218

12. McKinley, R.: Classical categories and deep inference. In: Structures and Deduction 2005 (Satellite Workshop of ICALP'05). (2005)

13. Lamarche, F.: Exploring the gap between linear and classical logic. Theory and Applications of Categories 18(18) (2007) 473-535

14. Došen, K., Petrić, Z.: Proof-Theoretical Coherence. KCL Publ., London (2004)

15. Gentzen, G.: Untersuchungen über das logische Schließen. I. Mathematische Zeitschrift 39 (1934) 176-210

16. Robinson, E.P.: Proof nets for classical logic. Journal of Logic and Computation 13 (2003) 777-797

17. Danos, V., Regnier, L.: The structure of multiplicatives. Annals of Mathematical Logic 28 (1989) 181-203

18. Buss, S.R.: The undecidability of $k$-provability. Annals of Pure and Applied Logic 53 (1991) 72-102

19. Lamarche, F., Straßburger, L.: Naming proofs in classical propositional logic. In Urzyczyn, P., ed.: TLCA'05. Volume 3461 of LNCS., Springer (2005) 246-261

20. Andrews, P.B.: Refutations by matings. IEEE Transactions on Computers C-25 (1976) 801-807

21. Bibel, W.: On matrices with connections. Journal of the ACM 28 (1981) 633-645

22. Guglielmi, A., Straßburger, L.: Non-commutativity and MELL in the calculus of structures. In Fribourg, L., ed.: Computer Science Logic, CSL 2001. Volume 2142 of LNCS., Springer-Verlag (2001) 54-68

23. Brünnler, K., Tiu, A.F.: A local system for classical logic. In Nieuwenhuis, R., Voronkov, A., eds.: LPAR 2001. Volume 2250 of LNAI., Springer (2001) 347-361

24. Straßburger, L.: From deep inference to proof nets via cut elimination. Journal of Logic and Computation (2009) To appear.

25. Guglielmi, A., Gundersen, T.: Normalisation control in deep inference via atomic flows. Logical Methods in Computer Science 4(1:9) (2008) 1-36 